\title{
Recusation du Juge dans la Procedure Civile : Cadre Juridique et Enjeux Actuels
}

\author{
Clément Shamashanga Minga ${ }^{1}$
}

\section{Résume}

La présente étude porte sur l'analyse du cadre juridique et des enjeux actuels de la récusation du juge dans la procédure civile congolaise. Elle démontre qu'à ce jour la récusation du juge est régie principalement par les dispositions des articles 49 à 59 de la loi organique portant organisation, fonctionnement et compétences des juridictions de l'ordre judiciaire. A ces dispositions s'ajoutent les dispositions des articles 36 à 42 de la loi du 16 octobre 2002 portant création, organisation et fonctionnement des tribunaux du travail ainsi que celles de l'article 16 de la loi du 03 juillet portant création, organisation et fonctionnement des tribunaux de commerce. Bien que reprenant les principales causes de récusation prévues par la majorité des législations des États, les dispositions régissant la récusation actuellement en République Démocratique du Congo ne couvrent pas les motifs qui donnent lieu à la récusation du juge. Ceci constitue une faiblesse en matière de la garantie de l'impartialité du juge qui est considérée comme la première exigence de la fonction de juger. Aussi, l'étude révèle que les demandes de récusation des juges aboutissent rarement à une décision devant les cours et tribunaux. Cette situation est due en premier lieu au faut que les juges mis en cause se déporter automatiquement sans attendre l'issue de la procédure et, en second lieu par le non-respect de la procédure par les justiciables ainsi que la difficulté pour ces derniers de prouver la preuve de partialité dans le chef du juge.

\begin{abstract}
This study focuses on the analysis of the legal framework and the current challenges of the judge's challenge in Congolese civil proceeding. It demonstrates that curently the challenge of the judge is governed mainly by the provisions of Articles 49 to 59 of the organic law on the organization, functioning and jurisdiction of the courts of the judiciary. To these provisions are added the provisions of Articles 36 to 42 of the Act of 16 October 2002 on the establishment, organization and functioning of labor courts, as well as those of Article 16 of the Act of 3 July establishing, organizing and operating Commercial courts. Although resuming the main causes of disqualification provided for by the majority of State legislation, the provisions governing recusation currently in the Democratic Republic of Congo do not cover the grounds giving rise to the disqualification of the judge. This is a weakness in the

1 Assistant à l'Institut Supérieur Pédagogique de Mweka (Kasaï), Avocat au Barreau de Matadi et Chercheur au Centre de Recherches et d'Etudes sur l'Etat de Droit en Afrique (CREEDA).Email : shamaclement@gmail.com.
\end{abstract}


guarantee of impartiality of the judge which is considered the first requirement of the judge function. As a result, the study reveals that challenges to judges are rarely resolved in the courts. This situation is primarily due to the need for the judges involved to deport automatically without waiting for the outcome of the proceedings and, secondly, the non-respect of the procedure by the litigants and the difficulty for them to prove evidence of bias on the part of the judge.

\section{Introduction}

Le droit au procès équitable, qui constitue un élément central et essentiel de l'Etat de droit ${ }^{2}$ ainsi que la pierre angulaire des procédures juridictionnelles ${ }^{3}$, implique entre autre le droit pour toute personne de voir sa cause être entendue équitablement et publiquement par des juges indépendant et impartiaux. L'indépendance des juges exige que ceux-ci soient affranchis de toute tutelle, de tout lien hiérarchique, non soumis à aucun ordre, aucune instruction ni influence afin de prendre leurs décisions uniquement sur la base du droit ${ }^{4}$. L'impartialité des juges, quant à elle, suppose que les juges ne puissent former leur conviction que sur des éléments objectifs, sans se laisser influencer par des arguments subjectifs ou des émotions personnelles ${ }^{5}$.

Ces deux exigences du procès équitable constituent, sans nul doute, non seulement le fondement de toute justice sur laquelle repose les fondations de l'Etat de droit, mais aussi le rempart contre l'éventuel arbitraire des juges ${ }^{6}$. Elles sont également à la base de la confiance des justiciables dans la justice et, portant, dans les juges qui la rendent. Il est, en effet, bien entendue que la justice ne peut être acceptée et regardée comme telle que si les juges qui la rendent sont indépendants et impartiaux. Et aucun citoyen ne soumettrait son affaire à l'appréciation des juges s'il s'avère que ces juges ne sont pas impartiaux.

Il est vrai que tous les juges sont présumés être indépendants et impartiaux ${ }^{7}$. Cependant, certaines situations peuvent amener les justiciables à douter de l'indépendance et de l'impartialité de certains juges appelés à connaître de leur affaire. Ainsi, il est permis à toute personne, partie dans un procès, qui a des bonnes raisons de craindre la partialité des juges

2 Sudre Fréderic, Droit européen et international des droits de l'homme, Paris, 2003, p.299.

3 GUINCHARD Serge et DEBARD Thierry (dir.), Lexique des termes juridiques, Paris, 2012, p.688.

4 Lire utilement le conseil consultatif des juges européens, Avis $\mathrm{n}^{\circ} 3$ sur les principes et règles régissant les impératifs professionnels applicables aux juges et en particulier la déontologie, les comportements incompatibles et impartialité, Strasbourg, 2002, p.2.

5 SHAMASHANGA MINGA Clément, « De la récusation des membres des cours constitutionnelles en Afrique francophone », In annuaire congolais de justice constitutionnelle, vol.2, 2017, p.57.

6 KAVUNDJA MANENO Télesphore, L'indépendance et l'impartialité du juge en droit comparé Belge, Français et de l'Afrique francophone, Thèse de doctorat en droit, Université de Louvain, 2005, p.6.

7 Betu Nzuji Achille, La récusation et la suspicion légitime en droit congolais : garantie d'une justice équitable, Kinshasa, 2013, p.8. 
appelé à connaître de son affaire de les récuser, c'est-à-dire de l'obliger à ne pas siéger comme magistrat et de se retirer ou de céder place à un autre juge ${ }^{8}$.

Cette possibilité est autorisée en principe dans toutes les procédures judiciaires. En effet, la législation congolaise autorise toute personne impliquée dans une procédure relative à la contestation de ses droits et obligations de caractère civil ou faisant l'objet d'une accusation en matière pénale de récuser tout juge dont elle peut avoir de raison de douter de sa capacité à rendre une décision impartiale. Cette possibilité s'applique en procédure pénale tout comme en procédure civile et administrative. Cependant, contrairement au droit français et belge qui prévoit des règles différentes en matière de récusation selon qu'il s'agit de la procédure pénale ou civile, le droit positif congolais applique mutatis mutandis la procédure de la récusation à la procédure pénale et à la procédure civile. En d'autres termes, des règles relatives à la récusation sont, en droit positif congolais communes à la procédure pénale et à la procédure civile. Et ces règles sont principalement régies par les articles 49 à 59 de la loi organique portant organisation, fonctionnement et compétences des juridictions de l'ordre judiciaire' .

$\mathrm{Au}$ - delà d'être un mécanisme de garantie du procès équitable en ce qu'elle tend à garantir le droit à un tribunal indépendant et impartial, la récusation, si elle est mal appliquée, risque de constituer une atteinte à l'honorabilité et à la dignité de la magistrature. Dans ce sens, Mukadi Boni et Katuala Kabakasha notent que la récusation des juges sans fondement peut porter atteinte à la considération et à l'honorabilité du juge ${ }^{10}$. D'où l'intérêt de présenter le cadre juridique et les enjeux de cette procédure enfin de rendre son application aisée. Pareille étude se justifie amplement dans le contexte congolais où l'observation empirique révèle que la procédure de récusation est parfois mal comprise tant par les justiciables que par les praticiens du droit. Il n'est pas inutile de révéler que certains justiciables et leurs représentants utilisent parfois la récusation comme moyen dilatoire pour bloquer ou retarder uniquement la procédure judiciaire. Par ailleurs, le déclenchement de la procédure de récusation par une partie au procès provoque parfois la colère du juge mise en cause. Tout ceci démontre à suffisance la mauvaise perception de la procédure de récusation par les justiciables et les acteurs de la justice.

Ainsi, cette étude aidera les praticiens du droit et les justiciables à connaitre l'importance de la procédure de récusation ainsi que les règles qui régissent cette procédure. Elle contribue dans cette logique à la bonne administration de la justice. Pour la réalisation de cette étude, nous avons effectué plusieurs descentes dans les greffes des Cours et tribunaux de la ville de Kinshasa pour se rendre compte de l'application de la procédure de récusation des juges principalement dans les procès civils. Aussi, nous avons eu plusieurs échanges avec les juges et les avocats ainsi que les greffiers pour avoir leurs points de vue la procédure de récusation telle qu'elle est appliquée devant les cours et tribunaux congolais. Par

8 Mukadi Boni et Katuala Kabakashala, Procédure civile, Kinshasa, 1999, p.99.

9 Journal officiel de la République Démocratique du Congo, $n^{\circ}$ Spécial du 4 mai 2013.

10 Mukdi Boni et Katuala Kabakashala, note 8., p.101. 
ailleurs, nous avons analysé un certain nombre des requetés en récusations ainsi que les arrêts de la Cour Suprême de Justice en matière de récusation. Cette méthode nous a permis de constater qu'il existe un divorce entre la pratique et ce que prévoit la loi en matière de récusation.

Cette précision importante, nous amène à présenter la notion de la récusation en droit en la distinguant des notions qui lui sont voisines avant de préciser les causes et la procédure de la récusation ainsi que l'enjeu de ce mécanisme.

\section{A. Notion sur la récusation}

Dans le langage du droit, la récusation traduit l'idée d'un acte permettant au plaideur de refuser d'être jugé en présence d'un ou des plusieurs juges qu'il estime impartiaux à son égard. Selon Gérard Cornu, la récusation est « un acte par lequel un plaideur refuse d'être jugé par ou en présence d'un magistrat ou un arbitre dont il conteste l'impartialité ${ }^{11}$.

Dans le même, Raymond Guillien et Jean Vincent définissent la récusation comme « une procédure par laquelle le plaideur demande que tel magistrat s'abstienne de siéger parce qu'il a des raisons de suspecter sa partialité à son égard $»^{12}$.Pour sa part, Pierre Okendembo Mulamba estime que la récusation est une « faculté reconnue à toute partie engagée dans un litige d'écarter la personne chargée d'y apporter la solution dès lors que celle-ci se trouve dans la condition où elle n'est pas en mesure de rendre sa décision en toute objectivité parce que son impartialité est mise en doute $»^{13}$.

A la suite de ces auteurs, nous pouvons dire que la récusation est un droit à la disposition de toute partie au procès de pouvoir obtenir qu'un membre d'une juridiction ne prenne part à l'affaire qui la concerne quand son impartialité semble incertaine. Autrement-dit, la récusation est un moyen reconnu à toute partie au procès de refuser que certains juges ne puissent connaître l'affaire qui la concerne dès lors qu'elle a des raisons de douter de leurs capacités de rendre une décision équitable. Il s'ensuit que le mécanisme de récusation permet de dessaisir un juge d'un dossier lorsque sa conduite, ses opinions, ou encore ses liens avec les parties ou l'objet du litige, suscitent un doute quant à son impartialité ${ }^{14}$.

La récusation constitue donc un incident qui intervient au cours de l'instance et à l'occasion duquel il est allégué qu'il existe des doutes quant à l'aptitude du juge à statuer de manière objective et impartiale sur le litige dont il est saisi ${ }^{15}$. Il s'agit là d'un principe universel et ancien fondé sur l'impossibilité pour le justiciable de se conformer à une décision

11 CORNU G., Vocabulaire juridique, Paris, 2011, p.859.

12 GUILLIEN R. et VICENT J.(dir), Lexique de termes juridiques, Paris, 2009, p..603.

13 Okendembo Mulamba P., Des procédures de récusation et de suspicion légitime en droit congolais, Kinshasa, 2012, p.14.

14 GUINCHARD Serge, Méga nouveau code de procédure civile, Paris, 2001, p.366.

15 Bendel Vasseur Stéphanie, «La procédure de récusation et de renvoi pour suspicion légitime en matière pénale ", Bulletin d'information de la Cour de cassation, nº731, du 15 novembre 2010, p.6. 
de justice s'il est prouvé que le juge a agi non selon la justice et le droit, mais par intérêts faveur ou haine.

De ce point de vue, la récusation s'avère être l'un des mécanismes destinés à garantir l'indépendance et l'impartialité des juges. En effet, c'est pour garantir l'impartialité et l'équité du jugement qu'il est reconnu à toute partie au procès la possibilité de solliciter la mise à l'écart de tout juge dont elle a des motifs légitimes et sérieux de douter de sa neutralité à son égard. Ainsi définie, la récusation doit être différenciée des autres notions qui lui sont voisines.

\section{B. La récusation et les notions voisines}

La récusation telle que définie ci-dessus doit être distinguée des notions telles que la suspicion légitime et le déport qui sont aussi des mécanismes renforçant l'impartialité des juges.

\section{La récusation et la suspicion légitime}

La suspicion légitime est définie par la doctrine comme « un soupçon de partialité envers la juridiction saisie qui permet à la juridiction supérieure, à la demande d'une partie, de dessaisir la première et de renvoyer l'affaire à une autre juridiction de même nature si le soupçon est fondée $»^{16}$. En terme claire, la suspicion légitime est une possibilité reconnue à toute partie au procès, qui a des motifs sérieux de penser que les juges appelés à connaître son affaire ne sont pas en situation de se prononcer avec impartialité en raison de leurs tendances ou de leurs intérêts, de pouvoir demander que l'affaire soit renvoyée devant une autre juridiction ${ }^{17}$.

A travers la procédure de suspicion légitime, une partie cherche donc à obtenir la mise à l' écart de toute une juridiction de la connaissance d'une affaire la concernant. C'est pour cette raison que Pierre Okendembo Mulamba considère la suspicion légitime comme « la récusation collective des membres d'une juridiction soit par la partie au procès, soit par le Ministère Public $»^{18}$.

Il ressort de ce qui précède que la suspicion légitime se rapproche de la récusation dans la mesure où elle apparait comme mécanisme visant à écarter la juridiction saisie de l'affaire dans le but de garantir l'impartialité de la décision à intervenir. Cependant, il y a lieu de souligner que la suspicion légitime se distingue de la récusation à plusieurs égards. A ce sujet, la Cour suprême de justice a précisé que « le renvoi pour cause de suspicion légitime n'est pas à confondre avec la récusation de l'un ou des plusieurs juges en application de l'article $71,7^{\circ}$ de l'ordonnance $n^{\circ} 82-020$ du 31 mars 1982 portant code de l'organisation et de la compétence judiciaires. Le renvoi pour cause de suspicion suppose, en effet, qu'il y a

16 Соrnu G., note 11, p. 999.

17 OkendemboMulamba P., note 13, p.17.

18 OkendemboMulamba P., note 13, p.18. 
impossibilité de constituer le siège à la suite d'une récusation visant l'ensemble des membres d'une juridiction $»^{19}$. Dans le même sens, il sied de dire que la récusation et la suspicion légitime se différencient à plusieurs points.

D'abord, si la demande de la récusation, comme on va le voir plus loin, vise individuellement un membre d'une juridiction de la connaissance d'une affaire dont elle est saisi.

Ensuite, si la demande de la récusation d'un membre d'une juridiction est déposée au greffe de la même juridiction, la requête en renvoi pour suspicion légitime doit être introduite devant une juridiction de rang supérieur à celle suspectée qui, à son tour, peut renvoyer, si elle trouve la demande fondée, la connaissance de l'affaire à une autre juridiction de même rang que la juridiction suspectée ${ }^{20}$. A ce jour, le tribunal de grande instance est compétent pour connaitre de toute demande en renvoi pour suspicion légitime contre le tribunal de paix de son ressort; la cour d'appel est compétente pour statuer sur la requête en renvoi pour suspicion légitime contre un tribunal de grande instance; et la cour de cassation est quant à elle compétente pour connaitre la demande en renvoi contre une cour d'appel.

Enfin, si la procédure de suspicion légitime n'est pas d'application dans un contexte où il n'y a pas de juridiction supérieure à celle suspectée comme le cas de la Cour de Cassation et du Conseil d'Etat, la récusation d'un juge est possible devant toute juridiction. La procédure de renvoi pour suspicion légitime contre la cour de cassation et le conseil d'Etat est impossible à cause non seulement de l'inexistence d'une juridiction supérieure à celles-ci pouvant ordonner le renvoi, mais aussi faute d'une autre juridiction de même rang et de même degré auprès de laquelle l'affaire puisse être renvoyée pour son examen en toute impartialité $^{21}$. Ceci dit, il convient à présent de distinguer la récusation et le déport.

\section{La récusation et le déport}

Le déport consiste pour le juge de s'abstenir de connaître l'affaire dont il est saisi pour motif de conscience ou parce qu'il suppose en sa personne une cause de récusation ${ }^{22}$. Le déport serait, selon Pierre Okendembo « une décision par laquelle un juge renonce spontanément à connaître le procès soit parce qu'il existe une cause de récusation en sa personne, soit parce qu'il y a pour lui un motif de conscience rendant souhaitable son abstention $»^{23}$. Il s'agit là d'une obligation légale et déontologique. En effet, la loi impose au juge de s'abstenir de siéger chaque fois qu'il existe une cause de récusation en sa personne.

Il ressort que le déport est un devoir pour le juge de s'abstenir de connaître une affaire dont il est saisie chaque fois que son impartialité pourrait être mise en cause. Le juge qui

19 Dibunda Kabuinji Mpumbuambuji, Répertoire général de jurisprudence de la Cour suprême de Justice de 1969 à 1985, Kinshasa, 1990, p.200.

20 Kavundja ManenoTélesphore, note 6, p.533.

21 Nganda Fumabo, L'audience judiciaire, Kinshasa, 2013, p.180.

22 Luzolo Bambi Lessa, Manuel de procédure pénale, Kinshasa, 2011, p.102.

23 OkendemboMulamba P., note 6, p.17. 
refuserait de se déporter alors qu'il sait qu'il est dans une situation qui mettra en doute son impartialité peut faire l'objet des poursuites disciplinaires. Cette précision permet de souligner qu'à la différence de la récusation, le déport est une décision volontaire et unilatérale d'un juge.

La distinction de la récusation avec les notions voisines conduit à analyser le cadre juridique de la récusation en République Démocratique du Congo.

\section{Le cadre juridique de la récusation}

La récusation des juges est principalement régie actuellement, pour les procédures pénales et civiles, par les dispositions des articles 49 à 59 de la loi organique $\mathrm{n}^{\circ} 13 / 011 \mathrm{~B} \mathrm{du} 11$ Avril 2013 portant organisation, fonctionnement et compétences des juridictions de l'ordre judiciaire. Ce sont ces dispositions qui consacrent non seulement les causes de la récusation, mais aussi la procédure à suivre pour déclencher cette demande. L'analyse de ces dispositions démontre que le législateur organique du 11avril 2013 a reconduit intégralement les dispositions des articles 71 à 73 de l'ancien code d'organisation et compétences judiciaires.

Par ailleurs, bien que reprenant les causes de récusation instituées par la majorité des législations, la loi organique sur l'organisation, fonctionnement et compétences des juridictions de l'ordre judiciaire reste lacunaire en ce qu'elle n'épuise pas toute les causes qui peuvent amener un justiciable à douter de l'impartialité des juges. au-delà des dispositions de la loi organique précitée, il faut ajouter les dispositions des articles 36 à 42 de la loi $n$ ${ }^{\circ} 016 / 2002$ du 16 octobre 2002 portant création, organisation et fonctionnement des tribunaux du travail ${ }^{24}$ ainsi que celles de l'article 16 de la loi $n^{\circ} 002 / 2001$ du 03 juillet 2001 portant création, organisation et fonctionnement des tribunaux de commerce ${ }^{25}$.

La précision du cadre juridique régissant la récusation autorise à préciser le fondement de ce mécanisme.

\section{Fondement de la procédure de récusation : la garantie de l'exigence d'impartialité}

La procédure de récusation est vue par une opinion comme portant atteinte à la considération et à l'honneur de la magistrature dans la mesure où cette dernière est censée être indépendante et impartiale. Une autre opinion la considère même comme un simple dilatoire destiné à retarder la procédure judiciaire et à empêcher la manifestation de la vérité. Il convient de relever que loin d'être considéré comme un moyen dilatoire destiné à retarder le cours normal d'une procédure judiciaire, la récusation doit être regarder comme un moyen destiné à garantir l'impartialité du juge qui est un droit fondamental pour tout citoyen et une obligation professionnelle pour le juge. En effet, plusieurs instruments juri- 
diques reconnaissent à toute personne le droit à ce que sa cause soit entendue équitablement par un tribunal indépendant et impartial ${ }^{26}$.

Par ailleurs, l'impartialité constitue un devoir pour tout juge. Dans ce sens, Serge Guinchard et Thierry Debard soulignent que l'impartialité est une exigence déontologique et éthique : le juge doit bannir tout a priori, excluant pareillement faveur et préférence, préjugé et prévention, ne céder à aucune influence de quelque source qu'elle soit; son obligation première est de tenir la balance égale entre les parties et de départager les prétentions en conflit uniquement par préférence au droit, à l'équité, à la justice, sans considération $»^{27}$.

L'obligation d'impartialité impose au juge la charge de conduire le procès dans les conditions offrant à chaque partie la possibilité de défendre ses intérêts équitablement ${ }^{28}$. Le juge se doit de garantir aux parties qu'il se déterminer en science et conscience dans le respect du droit positif, sans être limité a priori dans sa capacité de jugement de l'affaire par ses opinions personnelles ou ses relations personnelles ou avec qui il entretient des intérêts patrimoniaux ${ }^{29}$.

Autrement, l'obligation d'impartialité impose au juge le devoir de ne pas céder, ni à la tentation du corporatisme, ni aux influences de son milieu, de ses conceptions intimes, encore moins à la démagogie. Il va sans dire que dans sa mission de dire le droit, le juge n'a autre guide que la loi et ne pourra fonder sa décision que sur le droit et non sur autres considérations subjectives.

Au demeurant, pour garantir l'impartialité du juge, la loi a mis en place plusieurs mécanismes dont la récusation. C'est dans le but de garantir le droit au tribunal l'impartial qu'il a été institué le mécanisme de récusation. La récusation constitue une mesure visant à prévenir le risque de partialité du juge ${ }^{30}$. Ce mécanisme est donc destiné à garantir la bonne administration de la justice. Il ne peut donc être utilisé à des fins autres que la prévention contre le risque de partialité. C'est pour cette raison d'ailleurs que la loi fixe elle-même les causes de la récusation qu'il convient d'analyser à présent.

\section{E. Les causes de récusation}

Il est bien entendu, que la récusation pour être acceptée doit être fondée sur les motifs clairs basés sur des éléments concrets. Et ces motifs sont limitativement énumérés par la loi. A ce sujet, l'article 49 de la loi organique n ${ }^{\circ} 13 / 011-\mathrm{B}$ du 11 avril 2013 portant fonctionnement et

26 Voir les articles 10 de la déclaration universelle des droits de l'homme, 14 du pacte international relatif aux droits civils et politiques, et 7 de la charte africaine des droits de l'homme et des peuples.

27 Guinchard Serge et Debard Thierry, note 3, p. 453.

28 Lestrade Eric, Les principes directeurs du procès dans la jurisprudence du conseil constitutionnel, Thèse de doctorat en droit, Université Montesquieu Bordeaux IV, 2013, p.263.

29 Bendel Vasseur Stéphanie, note 15, p.9.

30 KavundjaManenoTélesphore, note 6, p.573. 
compétences des juridictions de l'ordre judiciaire énumère huit causes de récusation aux termes de cette disposition, tout juge peut être récusé pour l'une des causes suivantes :

- Si lui ou son conjoint à un intérêt personnel dans l'affaire;

- Si lui ou son conjoint est parent ou allié en ligne directe, soit en ligne collatérale jusqu'au troisième degré inclusivement de l'une des parties, de son avocat ou de son mandataire;

- S'il existe une amitié entre lui et l'une des parties;

- S'il existe des liens de dépendance étroite à titre de domestique, de serviteur ou d'employé entre lui et l'une des parties;

- S'il existe une inimitié entre lui et l'une des parties;

- S'il a déjà donné son avis dans l'affaire;

- S'il est déjà intervenu dans l'affaire en qualité de juge, de témoin, d'interprète, d'expert, d'agent de l'administration d'avocat ou de défenseur judiciaire;

- S'il est déjà intervenu dans l'affaire en qualité d'officier de police judiciaire ou d'officier du Ministère Public.

Il sied de dire que les causes de récusation telle que énumérées par la loi se basent sur les données objectives notamment la parenté, les relations amicales ou inamicales, l'existence d'autres procédures judiciaires où le juge est intervenu, etc. Aussi, l'énumération de ces causes par le législateur permette aux juges et parties de mesurer si la récusation se justifie dans un dossier. L'analyse en détail de ces causes permet une bonne utilisation du mécanisme de récusation par les justiciables et leurs représentants.

\section{Le juge ou son conjoint a un intérêt personnel quelconque dans l'affaire}

Il est bien entendu que le juge qui a intérêt direct ou indirect dans l'affaire peut être tenté de donner raison à l'une des parties au procès afin d'obtenir une faveur en retour. Il est de ce fait dans une situation inconfortable pour rendre une décision impartiale. Le fait pour le juge ou son conjoint d'avoir un intérêt personnel dans l'affaire fait naitre un doute quant à son impartialité. C'est pourquoi la loi autorise le justiciable, qui a la preuve que le juge a intérêt dans le dossier, de solliciter son remplacement un autre juge.

\section{Le juge ou son conjoint est parent ou allié de l'une des parties ou de son avocat ou de son mandataire}

Le juge qui a des liens des parentés avec l'une des parties au procès est dans une position qui met en doute son impartialité. Pareil juge ne peut trancher le litige en toute objectivité; Il doit être récusé. La récusation dans ce cas permet d'éviter le juge puisse favoriser sa famille ou celle de son conjoint. Il est serait judicieux de retenir que la cause de récusation demeure même en cas du décès du conjoint. L'expression « conjoint » du juge doit être étendue au concubin de ce juge. 


\section{Le juge est ami de l'une des parties}

L'existence des liens d'amitié entre le juge et l'une des parties au procès peut compromettre sérieusement l'impartialité de ce juge qui pourrait être amené à donner raison à son ami. De même, le juge qui a une connaissance personnelle de l'une des parties ne peut se prévaloir de son impartialité objective. Le juge devrait être récusé même lorsqu'il s'avère qu'il a des relations d'amitié avec les représentants de l'une des parties au procès. Il est regrettable de constater le législateur n'ait pas intégré de cette donne dans la cause de récusation en examen.

\section{L'existence des liens de dépendance ou de subordination entre le juge et l'une des parties}

Il est clair que le fait pour le juge d'avoir des liens de dépendance étroite à titre de domestique, de serviteur ou d'employé avec l'une des parties peut légitimement amener l'autre partie à douter de son impartialité. Il s'agit d'une situation inconfortable qui altère la confiance des justiciables dans le juge. Pour cette raison, il doit être écarté de la connaissance de la cause dont il est saisi.

\section{La connaissance antérieure de l'affaire par le juge}

La récusation d'un juge peut être demandée si ce juge a connu l'affaire comme ministère public, arbitre ou avocat, ou s'il est intervenu comme témoins. Cette cause de récusation cherche à éviter que le juge ait déjà une opinion préconçue du litige du fait d'avoir précédemment pris connaissance de ce litige. Aussi, le juge qui a connu l'affaire au premier degré ne peut être appelé à connaitre la même affaire au second degré.

Dans ce sens, la Cour suprême de justice a jugé : « doit être cassé l'arrêt d'une cour d'appel signé par un conseiller qui avait déjà siégé dans la même affaire quand il était juge au premier degré et que dans ces conditions, il ne s'est pas déporté en application de l'article 77 du code de l'organisation et de la compétence judiciaires $»^{31}$. Aussi, il a été jugé qu' « un magistrat du siège ayant instruit et jugé la cause avant poursuite judiciaire sur le plan discipline, devait se récuser, vu qu'il avait connu cette affaire devant une autre juridiction et partant, la décision est à annuler $»^{32}$.

\section{Le juge a déjà donné son avis dans l'affaire}

Le juge qui a déjà donné son avis dans une affaire dont il est appelé à trancher est dans une position qui fait douter de son impartialité dans la mesure où il peut déjà se faire une opi-

31 Arrêt R.P.60 du 2 février 1972, In Bulletin des arrêts de la Cour suprême de justice, année 1972, Kinshasa, 1973, p.16.

32 Dibunda Kabuinji Mpumbuambuji, note 19, p.198. 
nion. Il doit dans ces conditions se déporter faute de quoi il peut être récusé par une partie qui a intérêt. L'avis dont il est question peut être verbal ou écrit. La Cour suprême de justice a jugé que « ne peuvent être considérés comme avis susceptible d'être invoqué comme cause de récusation ou de déport, au sens de l'article 71 à 78 du code de l'organisation et de la compétence judiciaires, le fait pour un juge d'avertir les parties des moyens qui paraissent, lors d'une instruction à l'audience, pouvoir être soulevé d'office et de les inviter à présenter les observations soit immédiatement soit dans le délai qu'il fixe $»^{33}$.Aussi, le fait pour le juge d'avoir rendu un jugement avant dire droit qui ne préjuge pas le fond ne peut être considéré comme un avis qui entraine sa récusation.

Au demeurant, il y a lieu de souligner que l'énumération limitative des causes de la récusation par la loi ne permet pas de couvrir toutes les circonstances qui risquent de porter atteinte à l'impartialité du juge. Au fait, le caractère limitatif de l'énumération de la récusation ne semble pas de nature à prendre en charge tous les cas qui peuvent créer de doute sur la neutralité du juge. Il existe en effet, bien des situations qui, selon les circonstances peuvent susciter la crainte de partialité.

La récusation peut être sollicitée la crainte de partialité. Elle peut tout de même être sollicitée lorsque les opinions, prises de position et la manière dont le juge conduit le procès soulèvent de doute sur son impartialité. Autrement dit, la façon dont le juge conduit le débat peut donner de même une apparence de partialité. D' ailleurs, dans la pratique, la majorité des demandes de récusation des juges sont justifiées par la crainte partialité provenant de la manière dont les jugent orientent les débats ou de leurs prises de position.

Par exemple, dans une lettre de récusation d'un juge adressée au premier président de la Cour d'appel de la Gombe en date du 15 juin 2016 par l'ABC DE CONSTRUCTION SARL, l'une des parties au procès sous RCA 32.731, on peut lire que la partie précitée récuse l'un des conseillers appelés à connaitre son affaire, en occurrence, le président de la chambre, au motif que ce dernier, sans vérifier la saisine de la cour, a décidé de mettre de côté ladite affaire afin qu'elle soit plaidée en violation des us du prétoire et de la loi en matière civile. Selon le récusant, l'attitude du président de la chambre crée de doute quant à l'impartialité. C'est pour cette raison qu'il récuse le juge précité ${ }^{34}$.

La même raison a été évoquée par un justiciable pour justifier la récusation d'un juge sous RP. 12576/12674 pendant devant le tribunal de paix de Kinkole. En effet, dans une lettre adressée au président du tribunal de paix de Kinkole en date du 22 mai 2018 et réceptionnée la même date au greffe, monsieur Bibi Azieme, l'une des parties au procès, récuse l'un des juges appelés à connaitre son affaire au motif que ce juge oriente les débats en faveur de l'autre partie ${ }^{35}$.

33 Dibunda Kabuinji Mpumbuambuji, note 19, p.198.

34 Lettre de récusation du président de chambre adressée au premier président de la cour d'appel de la Gombe par la partie ABC DE CONSTRUCTION SARL sous RCA 32.731, consultée le 15 Aout 2018 au Greffe de la Cour.

35 Lettre de récusation du juge adressée au président de tribunal de paix de Kinkole par monsieur BIBI AZIEME, consultée au greffe du tribunal en date du 13 Aout 2018. 
Comme on peut le constater, les circonstances qui peuvent amener un justiciable à douter de l'impartialité du juge sont nombreuses et ne sont pas toutes reprises par la loi. C'est ainsi que les pays comme le Canada, dans le but de renforcer les mécanismes assurant l'impartialité du juge, ont institué la crainte raisonnable de partialité du juge comme cause de récusation. L'on peut donc relever que les dispositions de l'article 49 de la loi organique sur les juridictions de l'ordre judiciaire n'énumèrent pas toutes les causes qui peuvent donner lieu à la récusation.

Il s'agit là d'une faiblesse dans la protection de l'impartialité du juge. Cette faiblesse a été corrigée par le législateur par la loi organique $\mathrm{n}^{\circ} 16 / 027$ du 15 octobre 2016 portant organisation, compétences et fonctionnement des juridictions de l'ordre administratif ${ }^{36}$ qui, tout en reprenant les causes communes de la récusation, ajoute au point 8 de son article 346 que tout juge peut être récusé s'il existe dans son chef un ensemble des circonstances qui montrent qu'il ne présente pas les garanties d'impartialité.

Cette disposition a l'avantage d'élargir les motifs qui peuvent fonder une partie à initier une requête en récusation. Sur base de cette disposition, la récusation d'un juge peut être demandée par une partie au procès même à l'absence des relations de parenté ou d'amitié entre le juge et l'une des parties. Il suffit que le requérant démontre qu'il a une crainte raisonnable de partialité du juge à son égard. L'article 49 de la loi organique relative aux juridictions de l'ordre judiciaire doit être adaptée pour intégrer d'autres causes de récusation dans l'optique de renforcer l'impartialité du juge. La récusation doit être faite selon la procédure présente par la loi.

\section{F. La procédure de la récusation}

La récusation en tant qu'incident soulevé par l'une des parties au cours d'une instance dans le but de faire écarter un juge qu'elle suspecte de partialité doit être initiée conformément à la procédure prévu à cet effet par le législateur. Ainsi dans le présent point, il est question de présenter les personnes qui peuvent récuser et celles contre qui la récusation peut initier avant de voir la forme dans laquelle peut être formé une demande en récusation.

\section{Les titulaires du droit de récusation}

Les titulaires du droit de récusation sont énumérés par la loi. Il s'agit de toute partie au procès. Il s'ensuit que le droit de solliciter la récusation appartient au demandeur, au défendeur, au civilement responsable, à l'intervenant volontaire ou forcée. Mais la question que l'on peut se poser est seule de savoir si qualité de partie au procès suffit pour solliciter la récusation d'un juge. En réponse à cette question, il sied de dire qu'en plus de la qualité de partie au procès, le demandeur en récusation doit avoir l'intérêt en démontrant que ses intérêts risquent d'être compromis suite à la partialité avérée du juge. Cette précision nous permet 
de souligner qu'en procès civil, le ministère public ne peut récuser un juge que s'il intervient en qualité de partie principale. Aussi, puisque le récusant peut se voir parfois être condamné au payement des dommages et intérêts en cas du rejet de son action de récusation surtout en appel, il est préférable que lorsque la demande de récusation est initiée par un avocat pour le compte de son client que cet avocat soit muni d'une procuration spéciale. Il est regrettable que le législateur n'ait pas prévu cette obligation.

La détermination de titulaire du droit de récusation autorise à soutenir que les personnes qui n'ont pas qualité de partie au procès ne peuvent initier la procédure de récusation d'un juge, il en est ainsi des témoins, des renseignant, des experts, etc.

\section{Les personnes récusables}

Il sied de noter que la récusation ne peut être dirigée contre tout juge. Elle doit être initiée contre les juges appelés à connaître l'affaire. Ainsi, toute action dirigée contre tout juge de la même juridiction non chargé de la solution du litige doit être déclarée contre le greffier d'audience, car n'étant pas chargé de donner la solution du litige en instance.

Partant, il y a lieu d'indiquer que la procédure de récusation s'applique aussi aux jugesassesseurs des tribunaux du travai ${ }^{37}$ et aux juges consulaires des tribunaux de commerce. A ce sujet, l'article 36 de la loi sur les tribunaux du travail ${ }^{38}$ dispose que tout juge assesseur peut être récusé pour l'une des causes énumérées limitativement ci-après: si lui ou son conjoint a un intérêt personnel quelconque au litige; si lui ou son conjoint est parent ou allié de l'une des parties jusqu'au second degré inclusivement; si dans l'année qui a précédé la récusation, il y a eu une action judiciaire civile ou pénale contre lui ou son conjoint et l'une des parties; s'il a donné un avis écrit sur le litige; s'il est employeur ou travailleur de l'une des parties. L'on doit tout de suite

Aussi, l'article 16 de la loi relative aux tribunaux de commerce ${ }^{39}$ autorise la récusation des juges consulaires pour les causes de récusation de droit commun.

$\mathrm{Au}$ demeurant, il sied de souligner qu'il existe actuellement un débat sur la récusabilité du président de la juridiction lorsqu'il statue en application de l'article 49 de l'Acte uniforme de l'OHADA portant procédures simplifiées de recouvrement des créances et des voies d'exécutions. Cette disposition dispose que la juridiction compétente pour statuer sur tout litige ou toute demande relative à une mesure d'exécution forcée ou à une saisie conservatoire est le président de la juridiction statuant en matière d'urgence ou le magistrat délégué par lui. Ainsi, l'on se demande si le président de la juridiction susvisée est susceptible de la procédure de récusation lorsqu'il intervient comme juridiction compétente pour statuer sur

37 Mukadi Boni, Droit du travail, Bruxelles, 2008, p.687.

38 Loi $n^{\circ} 016 / 2002$ du 16 octobre 2002 portant création, organisation et fonctionnement des tribunaux du travail, note 24.

39 Loi $n^{\circ} 002 / 2001$ du 03 juillet 2002 portant création, organisation et fonctionnement des tribunaux de commerce, note 25. 
tout litige ou toute demande relative à une mesure d'exécution forcée ou à une saisie conservation.

A cette question, il faut dire que conformément à l'article 49 de la loi organique relative aux juridictions de l'ordre judiciaire le président de la juridiction précitée est récusable lorsqu'il existe dans son chef une cause de récusation. Cependant, il sied de révéler que la matérialisation de cette procédure dans ce cas n'est pas facile. C'est ainsi que Tony Muaba Kazadi considérant le président de la juridiction susvisée comme une véritable juridiction préfère qu'il fasse non pas l'objet de la procédure de récusation, mais plutôt de renvoi de juridiction $^{40}$. Bien que proposant une solution capable de résoudre actuellement la question, la solution proposée risque de poser aussi de problème en l'absence des dispositions légales. C'est pourquoi nous proposons la reformer législative dans le but de permettre dans une telle hypothèse que la demande de récusation du président statuant conformément à l'article 49 de l'acte uniforme précité soit adressée au premier président de la Cour d'appel du ressort.

\section{Conditions de recevabilité de la requête en récusation}

La lecture des dispositions légales sur la récusation autorise à soutenir que la recevabilité de la demande de récusation est liée au respect des conditions tenant au moment et de la forme de la présentation de cette demande.

\section{Le moment de la demande en récusation}

En application de l'article 50 de la loi organique sur les juridictions de l'ordre judiciaire, la partie qui veut récuser un juge le fait, sous peine d'irrecevabilité, dès qu'il a connaissance de la cause de récusation ${ }^{41}$ et au plus tard avant la clôture des débats. Deux observations méritent d'être faits. Premièrement, il sied de noter que la demande de récusation doit être impérativement formée avant la clôture des débats. Il s'ensuit que la demande de récusation ne peut en aucun cas être formée après la prise de l'affaire en délibérée. Serait donc irrecevable une demande de récusation formée après la clôture des débats. Il en découle que dès que les débats son clos, la récusation n'est plus possible ${ }^{42}$.

Dans le même sens, la jurisprudence précise : « Aucun délai n'étant prescrit, la récusation doit néanmoins être proposée in limine litis. En effet la partie peut renoncer à invoquer les griefs qu'elle aurait contre le magistrat, et elle est censée y avoir renoncé si elle engage

40 Muaba Kazadi Tony, « la récusation du juge président de la juridiction compétente instituée par l'article 49 de l'AUPSRVE », in magasine JuriAfrique du 26 septembre 2016.

41 Ibula Tshatshila Amédé, Droit de l'organisation et de la compétence judiciaires, Kinshasa, 2015, p.92.

42 BETU NZUJI Achille, note 7, p.46. 
le débat au fond sans les avoirs fait valoir. Elle ne pourrait les proposer en cours d'instance que si elle établissait les avoirs ignorés $»^{43}$.

Abondant dans le même sens, Pierre Okendembo Mulamba relève que « la récusation étant incident dilatoire, qui suspend le cours normal du procès, doit être soulevé in limine litis et le juge n'est pas permis de le joindre au fond de l'affaire $»^{44}$.

Il ressort de ce qui précède que si donc le juge n'est pas récusé avant la clôture des débats, le récusant n'aura plus l'occasion de soulever son incident, qui ne fera plus l'objet d'un débat contradictoire à l'audience. Ainsi, il est permis de soutenir que la déclaration avant la clôture des débats est une condition de recevabilité de l'action, quelles qu'en soient les causes. Mais il faut ajouter que la recevabilité de la demande en récusation est encore liée à la forme de la présentation de la requête.

\section{Modalité ou forme de la demande}

Selon la loi, celui qui veut récuser un juge le fait par une déclaration motivée et actée au greffe de la juridiction dont le juge mis en cause fait partie ${ }^{45}$. L'on peut déjà à nouveau souligner que la déclaration de récusation ne peut être écrite ou verbale. En plus, elle doit être motivée. Le récusant doit motiver sa demande de récusation en joignant parfois les pièces qui fonde sa crainte de partialité.

En d'autres termes, la demande de récusation doit être précise et motivée, et doit être accompagnée des pièces propres à la demande ou des justifications utiles. Le récusant doit mentionner le nom du juge mise en cause, il convient de préciser la déclaration de récusation faite par une partie ne suffit pas pour obliger le juge de se retirer. La déclaration est soumise à l'appréciation du tribunal conformément à la procédure prévue à cet effet par la loi.

Partant, l'observation empirique permet de relever que les justiciables respectent rarement la forme de déclaration de récusation prescrite par la loi. Au lieu de faire une déclaration de récusation au greffe comme dispose la loi, certains justiciables et leurs représentants préfèrent saisir directement le président de la juridiction dont fait partie le juge mis en cause. Cette pratique est en marge de la loi. La demande de récusation d'un juge doit être faite au greffe de la juridiction.

\section{Du déroulement de la procédure en récusation}

Une fois actée au greffe de la juridiction, la déclaration de récusation doit être notifiée au président de la juridiction ainsi qu'au juge mis en cause. Le juge mis en cause doit donner sa position par une déclaration écrite ou verbale de l'acte de récusation. Il faut dire que dès

43 LukooMusubao Ruffin, La jurisprudence congolaise en procédure civile, Kinshasa, 2009, p.322.

44 Okendembo Mulamba P., note 13, p.31.

45 Article 50 de la loi organique portant organisation, fonctionnement et compétences des juridictions de l'ordre judiciaire, note 9. 
qu'il est notifié de l'acte de récusation, le juge doit s'abstenir de poser les actes dans l'affaire jusqu'à ce qu'il soit statué sur la récusation. Le juge incriminé peut, s'il estime fondée la demande de récusation, se déporter. Dans le cas contraire, la juridiction à laquelle il appartient statuera sur la récusation, toutes affaires cessantes. Le juge mise en cause ne peut faire partie du siège appelé à statuer sur la récusation car l'on ne peut être juge et partie. La partie récusant doit être entendue par le tribunal.

Au demeurant, à l'issue de l'examen de la requête en récusation, deux solutions se présentent : la procédure de la récusation peut aboutir soit une décision du rejet, soit à une décision d'admission. Ainsi, si la récusation est admise, il sera procédé au remplacement, par le président de la juridiction, du juge récusé par un autre. Par ailleurs, si le tribunal rejette la récusation, le juge mis en cause sera ordonné à poursuivre l'instruction de la cause nonobstant l'appel.

Il est bien entendu qu'en cas du rejet de sa demande de récusation, le récusant a la possibilité de faire appel de la décision du rejet. Ainsi, si la juridiction d'appel confirme le jugement rejetant la récusation, elle peut condamner le récusant à une amende de cinq cent mille francs congolais sans préjudices des dommages et intérêts envers le juge mis en cause.

Par ailleurs, en cas d'infirmation du jugement rejetant la récusation au premier degré, le juge d'appel annule toute la procédure du premier degré qui en est la suite et renvoie les parties devant le même tribunal voisin du même degré sans préjudice de l'action disciplinaire. Il s'avère que pour éviter d'être condamné à une amende prévue par la loi et au dommages-intérêts, le récusant doit avoir la preuve de son allégation.

$\mathrm{Au}$ demeurant, les recherches effectuées dans les différents greffes des cours et tribunaux permettent de relever que les demandes de récusation des juges aboutissent rarement à un jugement devant les juridictions congolaises. L'une des raisons de cette situation est que le juge mis en cause préfère se déporter avant même la mise en examen de la requête en récusation. L'on peut lire ceci dans une lettre adressée au premier président de la Cour d'appel de Kinshasa/Gombe en date du 16 mai 2011 par un conseiller de cette juridiction : «Monsieur le premier président, après avoir lu, pour avis la lettre vous adressée en date du 04 mai 2011 par le maitre Marcel Kayembe Tshipampa, relative à l'objet en marge, la composition Mwanga Penga Penga, Kalume dans la cause sous RCA26.511 dont je représente, estime, par élégance, se déporter, bien que les faits allégués soient totalement faux et non motifs de récusation $»^{46}$.

L'attitude de juge de se déporter automatique chaque fois qu'il est récusé est encouragée par certains juristes qui estiment que le juge qui s'accroche à un dossier alors qu'il est récusé démontre qu'il a intérêt personnel dans l'affaire. Achille Betu Nzuji ne dit pas autre chose lorsqu'il écrit : « pour son honneur, un magistrat récusé devrait se déporter s'il n’a

46 Lettre adressée au premier président de la cour d'appel de Kinshasa/Gombe par le président Mwanga Mulindia en date du 16 mai 2011, consultée au greffe de la cour le 15 Aout 2018. 
aucun intérêt personnel et particulier $\gg^{47}$. Pareil conseil risque de déstabiliser les juges dans la mesure où il encourage le justiciable à obtenir automatiquement le remplacement d'un juge sans motif valable. Dans l'optique de protéger les juges face aux menaces des justiciables, il est encourageant que la requête en récusation soit examiné par le tribunal et qu'il puisse aboutir à une décision. La crainte selon le juge récusé et dont la demande de récusation est déclarée non fondée risquerait de se venger dans sa décision ne se justifie pas dans la mesure où le jugement est dans la majorité de cas une œuvre d'une juridiction collégiale. $\mathrm{Au}$ demeurant, pour obtenir la récusation du juge le récusant doit apporter la preuve de la partialité du juge mise en cause.

\section{G. De la preuve de la partialité}

Pour être établi, la demande de récusation doit être fondée sur une preuve de partialité. Et pour établir la partialité du juge, il ne suffit pas de démontrer qu'un juge a certaines croyances, opinions ou préjudices. Il faut établir que ses affinités avec l'une des parties ou ses opinions lui empêchent de rendre une décision impartiale. D'ailleurs, la Cour suprême de justice estime que des simples apparences ne suffisent pas. C'est ainsi qu'elle considère que manque de pertinence, le moyen fondé sur la violation du principe de neutralité du juge imposé par la procédure accusatoire lorsque ce dernier a accrédité la thèse du défendeur; l'attitude du juge ne constitue pas, en effet, une violation de ce principe mais une souveraine appréciation des prétentions qu'une partie a exposées dans ces moyens de défense.

C'est ici où se pose le problème en matière de récusation. S'il est facile de prouver l'alliance ou la parenté du juge avec l'une des parties, il n'en est pas de même des autres causes. Le fardeau de la preuve en matière de récusation incombe au récusant.

Ce dernier doit avancer des éléments qui ont fait naitre en lui la crainte quant au manque d'impartialité des juges. L'on indiquer qu'il serait aberrant de soumettre la preuve en matière de récusation aux règles qui gouvernent la preuve en matière civile. En procédure de récusation, le récusant doit être libre de prouver que le juge mis en cause est dans une position qui suscite de doute quant à son impartialité. Dans le même sens, Achille BetuNzuji souligne que la partialité d'un juge doit être prouvées par toutes les voies de droit, notamment ses déclarations faites avant, pendant et après l'audience, la jonction au fond d'une exception visiblement fondée et à laquelle une décision devrait être rendue, l'orientation de l'instruction dans le sens favorable à une partie ${ }^{48}$.

Une partie de la doctrine enseigne qu'en matière récusation, il n'est pas bon de s'accrocher à la preuve. La crise de confiance suffise pour qu'un juge soit récusé. La Cour suprême de justice a plusieurs fois statué dans ce sens, en décidant que « lorsqu'une partie a

47 Betu Nzuji Achille, note 7, p.14.

48 BetuNzuji Achille, note 7, p.13. 
perdu confiance dans son juge naturel que lui a assigné la loi, il importe de renvoyer les parties devant un autre juge afin d'assurer une bonne administration de la justice $»^{49}$.

Il ressort de cette jurisprudence que pour une bonne administration de la justice, laquelle doit être rendue en dehors de toute pression, la demande récusation peut être accordée même si les faits dénoncés à l'encontre des juges mis en cause ne sont pas établis.

\section{Conclusion}

Par cette réflexion portant sur la récusation dans les procédures civiles congolaises, il nous a fallu éclairer le lecteur sur le cadre juridique et les enjeux actuels de la procédure de récusation des juges, considérée à juste titre comme mécanisme de garantie de droit à un tribunal indépendant et impartial et, partant, de la bonne administration de la justice.

Cette étude que révèle que les dispositions relatives à la procédure de récusation sont diversement interprétées tant par les justiciables que par les praticiens du droit dont les juges et les avocats. En effet, les descentes dans les différents greffes des cours et tribunaux ont permis de constater qu'il existe un écart profond entre ce que prévoit la loi sur la récusation et la pratique de celle-ci. Cet écart semble certainement dû à l'ignorance des règles qui gouvernent la procédure de récusation en droit congolais. Il est aujourd'hui difficile de trouver dans nos cours et tribunaux une décision sur la récusation. Cette situation est due au fait que certains juges mis en cause préfèrent se déporter automatiquement sans attendre qu'il soit statué sur le mérite ou le bienfondé de la demande en récusation. Aussi, la pratique révèle dans la plupart des cas, les justiciables ne respectent pas la procédure prévue par le législateur pour solliciter la récusation d'un juge. Cette situation est due aussi l'ignorance des règles qui régissent la procédure de la récusation des juges dans les procédures judiciaires congolaises.

Ainsi, elle propose la vulgarisation et la réforme des certaines dispositions qui régissent la procédure de récusation enfin de rendre aisée l'application et la compréhension de ce mécanisme dont le but est de garantir l'indépendance et l'impartialité du juge. Pareille mesure ne pourra que contribuer à l'amélioration de l'image de la justice congolaise.

\section{Bibliographie}

Loi organique $\mathrm{n}^{\circ} 13$ / portant organisation, fonctionnement et compétences des juridictions de l'ordre judiciaire, Journal officiel de la RDC, $\mathrm{N}^{\circ}$ spécial du 4 mai 2013.

Loi n⿳00/2001 du 03 juillet 2001 portant création, organisation et fonctionnement des tribunaux de commerce, Journal officiel, ${ }^{\circ} 14$ du 15 juillet 2001.

Loi $n^{\circ} 016 / 2002$ du 16 octobre 2002 portant création, organisation et fonctionnement des tribunaux du travail, Journal officiel, $n^{\circ}$ Spécial du 25 octobre 2002. 
Loi organique $\mathrm{n}^{\circ} 16 / 27$ du 15 octobre 2016 portant organisation, compétences et fonctionnement des juridictions de l'ordre administratif, Journal officiel, n Spécial du 18 octobre 2016.

SUDRE Fréderic, Droit européen et international des droits de l'homme, Paris, 2003. GUINCHARD Serge et DEBARD Thierry, Lexique des termes juridiques, Paris, 2012

SHAMASHANGA MINGA Clément, « De la récusation des membres des cours constitutionnelles en Afrique francophone », In Annuaire congolais de justice constitutionnelle, Vol.2, 2017.

KAVUNDJ MANENO Téléphone, L'indépendance et l'impartialité du juge en droit comparé Belge, Français et de l'Afrique francophone, Thèse de doctorat en droit, Université de Louvain, 2005.

BETU NZUJI Achille, La récusation et la suspicion légitime en droit congolais : garantie d'une justice équitable, Kinshasa, 1999.

MUKADI BONI et KATUALA KABAKASHALA, Procédure civile, Kinshasa, 2013.

CORNU G., Vocabulaire juridique, Paris, 2011.

GUILLIEN R. et VINCENT J., Lexique des termes juridiques, Paris, 2009.

GUINCHARD Serge, Méga nouveau code de procédure civile, Paris, 2001.

OKENDEMBO MULAMBA Pierre, Des procédures de récusation et suspicion légitime en droit congolais, Kinshasa, 2012.

BENDEL VASSEUR Stéphanie, "La procédure de récusation et de renvoi pour suspicion légitime en matière pénale ", Bulletin d'information de la Cour de Cassation n731 du 15 novembre 2010.

DIBUNDA KABUINDI MPUMBUAMBUJI, Répertoire général de jurisprudence de la Cour Suprême de Justice de 1969 à 1985, Kinshasa, 1990.

LUZOLO BAMBI Lessa, Manuel de procédure pénale, Kinshasa, 2011.

LESTRADE Eric, Les principes directeurs du procès dans la jurisprudence du Conseil constitutionnel, Thèse de doctorat en droit, Université Montesquieu Bordeaux IV, 2013.

IBULA TSHATSHILA Amedé, Droit de l'organisation et la compétence judiciaire, Kinshasa, 2015. LUKOO MUSUBAO Ruffin, La jurisprudence congolaise en procédure civile, Kinshasa, 2009. 